\title{
Économie des honneurs techniques et production de la qualité sous la Troisième République
} L'action de la Société d'encouragement à l'art et à l'industrie

\section{Stéphane Lembré}

\section{(2) OpenEdition Journals}

Édition électronique

URL : https://journals.openedition.org/artefact/9099

DOI : 10.4000/artefact.9099

ISSN : 2606-9245

Éditeur :

Association Artefact. Techniques histoire et sciences humaines, Presses universitaires du Midi

Édition imprimée

Date de publication : 11 septembre 2014

Pagination : 101-111

ISBN : 978-2-271-08150-6

ISSN : 2273-0753

\section{Référence électronique}

Stéphane Lembré, «Économie des honneurs techniques et production de la qualité sous la Troisième République », Artefact [En ligne], 2 | 2014, mis en ligne le 12 mai 2021, consulté le 24 août 2021. URL : http://journals.openedition.org/artefact/9099; DOI : https://doi.org/10.4000/artefact.9099

\section{cc) (D)}

Artefact, Techniques, histoire et sciences humaines est mise à disposition selon les termes de la Licence Creative Commons Attribution - Pas d'Utilisation Commerciale - Pas de Modification 4.0 International. 


\section{Économie des honneurs techniques et production de la qualité sous la Troisième République. L'action de la Société d'encouragement à l'art et à l'industrie}

Stéphane LEMBRÉ ${ }^{1}$

\section{Résumé}

Face au déclin réel ou présumé des industries d'art françaises, la Société d'encouragement à l'art et à l'industrie est fondée à la suite de l'Exposition universelle de 1889, à Paris. Sous l'impulsion des principaux responsables de l'éphémère ministère des Arts du gouvernement Gambetta, elle s'engage dans la promotion de la formation technique, dont la nécessité est reconnue: la mise en place d'un concours de composition décorative et de bourses d'apprentissage, puis la participation active aux expositions d'art industriel dans les années 1920, constituent ses principaux moyens d'action. Ils témoignent d'une pédagogie de la qualité. En encourageant, en récompensant et en participant à la définition de la qualité, la Société contribue à la définition d'une économie des honneurs de type méritocratique et assigne à la formation technique une fonction essentielle pour en conforter la légitimité.

Mots-clés : art, apprentissage, France, industrie, méritocratie, qualité.

\section{Abstract}

Because of the possible decline of the French industrial art, the Sociéte d'encouragement à l'art et à l'industrie was established after the World Fair of 1889 in Paris. Initiated by the heads for the short-lived ministry of the Arts, the Society members contributed to the implementation of an ornamental composition competition and student's grant for apprenticeship. Among its main actions, the Society was also very active in industrial art exhibitions in the 1920s. Such an evolution can be found particularly in the conjugated efforts to encourage, to reward and to take part in the definition of quality. Thus the 
Society contributed to the development of an economy of the honors. This meritocratic policy assigned to the technical training an important function.

Keywords: apprenticeship, art, France, industry, meritocracy, quality.

Dans l'affirmation de l'école républicaine en France, le rôle de la méritocratie est central pour justifier la construction de hiérarchies sociales entre des citoyens égaux. Par les distributions de prix, notamment, une véritable économie des honneurs, héritière d'une ingénierie constituée depuis le $\mathrm{xvIII}^{\mathrm{e}}$ siècle, caractérise le système éducatif². Si cette économie des honneurs et le fait de « décorer le mérite» sont «une technique de gestion sociale ${ }^{3} »$ antérieure à la Troisième République, quoique prolongée et amplifiée par les promoteurs de l'école républicaine, la plupart du temps, ce sont les récompenses distribuées dans les écoles primaires et secondaires qui ont été décrites, au risque de masquer la grande diversité des récompenses et des publics concernés. De même que les enseignements techniques sont restés longtemps à la marge du système scolaire français et des travaux des historiens, la place de ces dispositifs dans la promotion d'une culture du travail dans la République n'a guère suscité d'études.

Or, cette économie des honneurs techniques contribue à dessiner les contours de la qualité et du savoir-faire. Elle participe de la reconnaissance des besoins de formation technique en encourageant et récompensant la qualité d'un produit et $\mathrm{d}^{\prime}$ un travail ${ }^{4}$. Sous un angle méritocratique, elle fournit des prescriptions de qualité grâce à des discours et des prix «adaptés» à l'enseignement technique, dans un système éducatif étranger à toute fonction de promotion sociale collective. Pour mesurer plus précisément les origines, les modalités et les objectifs de cette forme d'encouragement, il est nécessaire d'étudier les liens entre la formation des producteurs, destinée à les doter de savoir-faire, et la qualification des produits, entendus comme des biens envisagés du point de vue de leur fabrication, de leur circulation, de leur consommation et des représentations qui leur sont associées. Il convient ainsi de saisir la construction d'une économie de la qualité au service de l'institutionnalisation de la formation technique ${ }^{5}$.

Les archives de la Société d'encouragement à l'art et à l'industrie, groupement de notables créé en 1889 et proche de l'administration des Beaux-Arts, se prêtent à cette problématique ${ }^{6}$. Conçue sur le modèle de la Société d'encouragement pour l'industrie nationale, créée en 1802 sous l'impulsion de Chaptal ${ }^{7}$, cette société place la formation technique au centre de son action pour enrayer le «déclin» français face à la concurrence dans le domaine des industries d'art. Dans ce «déclin», il est difficile de faire la part des difficultés réelles et de la dimension imaginaire, tant se propage cette réaction commune d'élites inquiètes devant l'émergence de nouveaux pays producteurs dans un contexte de «crise» mondiale. Que l'économie des honneurs constitue un levier d'action privilégié par la Société d'encouragement ne doit pas surprendre, car les expositions uni- 
verselles avaient déjà habitué à cette logique de l'émulation pacifique, voire pacificatrice - faut-il rappeler que, lors de l'Exposition universelle de Paris en 1889, plus de 33000 récompenses ont été distribuées pour 60000 exposants $^{8}$ ? Un tel ratio n'est pas isolé, bien qu'il ne dise rien de l'usage des prix et des récompenses. Ce qui, en revanche, paraît moins attendu dans le cas de cette Société tributaire d'un modèle défini plusieurs décennies plus tôt, c'est le choix d'une économie des honneurs techniques, récompensant ensemble la formation et la qualité à partir de jugements sur les produits. Les prix attribués sont importants pour la réputation des producteurs et pour les institutions de formation. À la différence des jurys pléthoriques constitués ponctuellement pour ces vitrines de la modernité que furent les expositions universelles, l'action de ce groupement au recrutement élitiste s'inscrit dans la durée. Elle prétend prescrire ce qu'est la qualité et ce que doit être la formation technique.

À la formation, envisagée comme un enjeu suffisamment important pour justifier la mise en œuvre d'un dispositif pédagogique, succède la récompense destinée à encourager la qualité. Les honneurs sont ainsi l'aiguillon imaginé par la Société pour encourager le développement de la formation. Ce système culmine avec un troisième temps logique, celui de l'exposition, que l'on peut assimiler à une pédagogie de l'objet de qualité: l'exposition doit faire connaître les normes de qualité et mettre en valeur des exemples jugés stimulants.

\section{Le temps de l'engagement: l'enjeu de la formation technique}

Alors que l'État républicain ne dispose pas, à la fin du xIX siècle, d'une politique d'enseignement technique comparable à celle qu'il développe avec volontarisme pour l'enseignement primaire, il multiplie en revanche les encouragements aux initiatives privées: faute de moyens financiers suffisants, les autorités s'appuient sur des groupements jugés plus compétents pour estimer les besoins de formation et les meilleurs moyens de les satisfaire, et s'évitent par la même occasion le reproche d'entorse au libéralisme. Par sa position à la charnière de l'enseignement et des activités économiques, la formation technique forme- rait un terrain où les «besoins» locaux et particuliers des entreprises doivent déterminer la mise en place des dispositifs de formation?. Les fondateurs de la Société d'encouragement à l'art et à l'industrie se posent en experts dans la définition de ces besoins, dans une période qui voit l'essor de l'enseignement technique, encouragé par la loi de 1880 sur les écoles manuelles d'apprentissage, puis la création en 1892 des écoles pratiques de commerce et d'industrie ${ }^{10}$. Comme l'Union centrale des arts décoratifs fondée en 1882, ils veulent concilier l'art et l'industrie, promouvoir le «bon goût» et la qualité française. Les statuts 
prévoient «le développement des arts appliqués à la décoration et à l'industrie par la collaboration de l'État et des initiatives privées, et le renouvellement des modèles des industries d'art par la coopération rationnelle de l'artiste, de l'industriel, de l'éditeur, de l'artisan, de $l^{\prime}$ ouvrier $\mathrm{d}^{\prime} \operatorname{art}^{11}{ }^{1}$ ».

L'intérêt pour l'art et l'industrie s'explique par les origines de la Société et celles de ses fondateurs, bons représentants de l'inquiétude qui se manifeste depuis le rapport du comte de Laborde à la suite de l'Exposition internationale de Londres de $1851^{12}$. La Société est créée dans le sillage de l'Exposition universelle de Paris organisée en $1889^{13}$. Le directeur des Beaux-Arts Gustave Larroumet y organise une classe réservée à l'enseignement du dessin décoratif et industriel, pourvue d'un jury réunissant artistes, fonctionnaires, amateurs d'art et industriels. Cette initiative s'appuie sur les vœux formulés lors de l'enquête organisée en 1881 par l'éphémère ministre des Arts Antonin Proust ${ }^{14}$, afin de procéder à un état des lieux des industries d'art, alors que le diagnostic d'une crise industrielle et artistique, partie prenante de la dépression économique des années 1880, se répand. Marius Vachon, enquêteur officiel, en confirmant les difficultés, exhorte à mettre «de l'art en tout, dans tout ce que nous faisons; apprenons à connaître et à aimer les belles choses, formons notre goût et élevons haut notre imagination. Soyons un peuple d'artistes en même temps qu'un peuple de marchands; cela n'est point inconciliable, à condition que les marchands soient des artistes ${ }^{15}$.»

Les fondateurs sont présentés dans les archives de la Société comme «un groupe d'amis» présidé par le joailler-horloger Gustave Sandoz. Celui-ci déploie une intense activité de propagandiste pour la production française: il est tour à tour fondateur du Comité d'initiative des expositions françaises à l'étranger (devenu le Comité français des expositions), de la Société d'économie industrielle et commerciale, promotrice de la Foire de Paris, président de la Section française à $l^{\prime}$ Exposition française de Barcelone de 1888, de l'Exposition française de Londres de 1890 et du Comité d'initiative de l'Exposition française de Moscou en 1891! À ses côtés, parmi la trentaine de membres fondateurs, on trouve des personnalités telles que le député Henry Maret, Gustave Larroumet ou encore Yves Guyot, alors ministre des Travaux publics. Par ses membres, la Société accompagne l'institutionnalisation d'un «système des Beaux-Arts ${ }^{16}$ " marqué par la création du conseil supérieur des Beaux-Arts (1875) et du bureau de l'enseignement au sein de la direction des Beaux-Arts (1878). Jusqu'en 1904, les quatre premiers présidents de la Société font partie de ce groupe fondateur. Les annuaires édités par la Société recensent 127 sociétaires en 1904 (et 184 en 1913), dont quatre ministres ou anciens ministres (André Lebon, Georges Leygues, Raymond Poincaré, Yves Guyot), neuf députés ou anciens députés; les banquiers Périer, l'imprimeur-éditeur Lahure, le parfumeur Aimé Guerlain, l'architecte Dutert, le statuaire Bartholdi donnent une idée du recrutement socialement élitiste.

Deux manifestations principales rythment l'activité de la jeune société: le concours annuel de composition décorative est créé en 1891 et, à partir de 1893, 
un second concours organisé entre les élèves des écoles de province octroie une bourse d'apprentissage dans une industrie d'art. Le premier concours de composition décorative porte sur une composition décorative susceptible de recevoir une application industrielle. Peuvent présenter leurs réalisations les élèves des écoles de dessin, de beauxarts, d'art décoratif et d'art industriel de Paris et des départements, «à l'exception toutefois de l'école de beaux-arts de Paris ${ }^{17}$ », que la Société semble ainsi exclure de cette orientation industrialiste. En 1892, il s'agit de la décoration de la reliure d'un livre. Lors du troisième concours annuel, 233 concurrents se présentent et le premier prix est attribué à un élève de l'École nationale des arts industriels de Roubaix, créée par une loi du 5 juillet 1881 pour devenir un fleuron de l'enseignement des industries d'art. Le concours pour l'obtention d'une bourse d'apprentissage organisé entre les écoles des départements assure au lauréat la jouissance pour trois ans d'une bourse de 1200 francs. Dès la seconde édition, en 1896, vingt et un candidats de onze écoles différentes sont admis à concourir. La bourse est attribuée à un élève de l'École des beaux-arts de Marseille, futur peintre décorateur. Cette bourse était revenue durant les années précédentes, à l'issue du premier concours en 1893, à un élève de l'École nationale de Roubaix, qui devient pupille de la Société, tandis que celle-ci recommande le candidat classé second, issu de la même école, pour une bourse de la municipalité roubaisienne $e^{18}$. La complémentarité institutionnelle souhaitée est patente, mais signale la limite des efforts financiers respectifs: la chambre de commerce de Roubaix, tout en approuvant l'action de la Société d'encouragement, renvoie aussi vers le conseil municipal la demande de subside. Après avoir profité de cet encouragement, le lauréat suit les cours d'une école normale de dessin à Paris, «et gagne aujourd'hui largement sa vie comme dessinateur en tissus et en papiers peints ${ }^{19}{ }^{\prime}$. Quelques membres actifs s'occupent de la question parfois épineuse du devenir des pupilles, par crainte qu'ils ne se destinent aux beauxarts plutôt qu'à la gravure industrielle et commerciale. Dans le contexte de la crise de l'apprentissage constamment déplorée, et en raison de son propre règlement, le concours peine à trouver son public, démontrant aux responsables de la Société la difficulté à passer de la théorie à la pratique en matière d'encouragement à la formation technique. Il n'en témoigne pas moins de la volonté de promouvoir une pédagogie de la qualité, jusque dans les prescriptions sur les méthodes d'enseignement du dessin dont certains membres nourrissent leurs rapports sur les concours.

Par leur fonction de soutien aux dispositifs de formation, les concours visent à réconcilier l'art et l'industrie, à articuler avec souplesse le goût du passé et le siècle de l'industrie. La récompense s'inscrit dans la continuité de cette action. 


\section{Le temps de la récompense : encourager la qualité}

La Société d'encouragement a l'ambition de hiérarchiser des égaux selon leur «talent» qu'elle se propose d'évaluer grâce aux compétences qu'elle réunit. Par ces dispositifs méritocratiques inspirés de l'univers scolaire, elle entend diffuser les idées de ses membres en matière d'union de l'art et de l'industrie.

Les encouragements prennent plusieurs formes. Financière, d'abord, avec des prix et des bourses. Symbolique, ensuite, sous forme de plaquettes ou de diplômes. En revanche, on ne trouve pas mention de boîtes d'outils, parfois distribuées dans les écoles d'enseignement technique, puis sous l'impulsion de la direction de l'enseignement technique dans les années 1920 et 1930. Pour afficher son soutien à des institutions et des projets proches de ses objectifs, la Société cherche en réalité les meilleurs moyens d'intervenir. En 1939, pour le concours annuel organisé au musée Galliéra, à l'occasion de l'exposition annuelle d'art décoratif moderne, les membres de la Société d'encouragement figurent pour moitié dans le jury. Quatre plaquettes de la Société, dues au sculpteur Oscar Roty, sont mises à la disposition de ce jury. Dix ans plus tôt, la Société avait entrepris de récapituler les sommes payées à titre de primes aux élèves des écoles de dessin, des beaux-arts, d'art décoratif et d'art industriel depuis le début des concours généraux annuels de composition décorative et industrielle. Sur près de quatre décennies, les écarts sont importants: les élèves d'écoles parisiennes ont reçu 40455 francs, suivent Marseille (9100 francs), Nice (8820 francs), Lyon (6095 francs), Roubaix (5850 francs). Au total, les écoles de trente et une villes différentes ont reçu des récompenses, quoique dix-neuf d'entre elles aient reçu moins de 1000 francs. La répartition des encouragements ne peut cacher leur modestie, même si cette distribution reflète l'intérêt porté localement aux concours.

L'ambition de développer l'art appliqué préside à ces actions. Les concours sont ainsi l'objet de rapports souvent précis et précieux quant aux liens entre formation, art et industrie et pour la définition de la qualité. À l'occasion du concours organisé au début de 1939 sous le patronage du ministre de l'Éducation nationale Jean Zay, le secrétaire général s'enorgueillit au nom de la Société d'avoir, depuis 1889, organisé «340 concours dont 48 de composition décorative et industrielle, lesquels ont réuni 18120 candidats dont 3129 ont été récompensés». Plutôt que ces chiffres - invérifiables -, la continuité de l'encouragement et les objectifs poursuivis méritent attention. Le programme soutenu par l'administration des beauxarts porte sur «un trophée sportif». Le rapport rédigé à l'issue du concours est éloquent:

"Cet élément décoratif qui tient une si grande place dans la vie populaire, et surtout dans celle des "jeunes", nous ont valu, en majorité jusqu'à ce jour, $\mathrm{d}$ 'innombrables et pitoyables laideurs. Très rares sont les œuvres intéressantes qu'il nous est donné de voir [...]. C'est pour réagir contre cette carence que la Société d'encouragement à l'art et à l'industrie avait donné ce programme 
capable d'enthousiasmer notre jeunesse française. Programme magnifique mais difficile. Éviter les écueils du banal ou de l'anecdotique, trouver une idée synthétique, simple et belle, cela est plus ardu qu'on ne le pense, le "grand art", suivant le mot de Racine, consistant à "faire quelque chose de rien".»

Le rapporteur évoque de grandes disparités entre les projets reçus et s'interroge face à certains "extrêmement médiocres » :

"Les causes de ces faiblesses indiscutables devraient être recherchées et s'il apparaissait qu'elles fussent dues à l'incompétence d'un professeur, un moyen devrait être recherché pour que la jeunesse studieuse n'en demeure pas trop longtemps la victime. Il est apparu en effet au jury qu'il était abusif de respecter certains intérêts particuliers quand ceux-ci handicapent l'avenir de centaines de jeunes gens et même $l^{\prime}$ avenir des industries locales ${ }^{20}$.»

Le concours devient ainsi un instrument prescriptif et de jugement des institutions de formation. Il renvoie aux catégories partagées par les membres du jury, et donc à leur définition de ce que devrait être la formation technique. Pour ce rapport, le bureau de la Société avait ajouté au concours une demande particulière à chaque école qui présentait des projets: "préciser combien elle consacrait d'heures à "l'Art appliqué" chaque mois". Les résultats sont peu exploitables faute de distinction claire entre le dessin et l'application mais prouvent la grande hétérogénéité des situations et la distinction difficile, voire impossible, entre l'enseignement «académique» et l'enseignement «appliqué» ${ }^{21}$. Le rapporteur conclut sur l'idée d'obliger les écoles, par l'intermédiaire des directions générales, à participer au concours, au nom de l'indispensable émulation.

Les objectifs de la Société la conduisent donc à définir la qualité. Ainsi, en 1906, pour le $16^{\mathrm{e}}$ concours général de composition décorative, le thème choisi est: «Une lanterne pour une antichambre». La description des projets attendus est précise:

«Cette lanterne, alimentée par le gaz ou l'électricité, mesurera au maximum $0 \mathrm{~m} 35$ de largeur sur $0 \mathrm{~m} 65$ de hauteur, y compris son attache, mais non compris la chaîne ou tige en suspension. Les matériaux utilisés pour la construction et l'ornementation de cette lanterne sont laissés au choix des concurrents. Il leur est seulement signalé que la lanterne, objet d'usage courant et sans prétention à la somptuosité, est destinée à distribuer le plus possible de lumière générale dans l'antichambre. [...]»

Derrière cette description, on repère la volonté d'introduire la qualité dans le quotidien ${ }^{22}$. La qualité est directement fonction de l'usage auquel l'objet est destiné et des matériaux qui seront employés.

Néanmoins, ces prescriptions trouvent parfois leurs limites. Dans son rapport sur le concours de composition décorative et industrielle de 1922, Henri Lefort déplore une "stagnation" des productions reçues et plaide pour l'abandon de la prétention uniformisatrice: 
«Il n'est que temps de s'entendre pour arrêter des programmes convenant aux écoles selon les besoins, les aptitudes et les ressources des régions. Existera-t-il jamais un Code rigide et des lois strictes applicables aux arts qui dérivent de l'enseignement du dessin et de la décoration? Assurément non, et d'ailleurs cette unification n'est pas à souhaiter. Elle serait plutôt à craindre. Car l'apprentissage en question comporte une inépuisable variété, des possibilités pour ainsi dire sans limites de compréhensions multiples, une succession de métamorphoses inédites, parfois opposées et sans cesse renaissantes.»

Conformément aux préconisations d'une adaptation locale des besoins de formation, sont ainsi mis en place des concours régionaux de composition décorative et industrielle, organisés par les comités régionaux des arts appliqués. Il est vrai que les normes de qualité énoncées par les membres de la Société d'encouragement ne sont pas toujours partagées. Les critiques sont fréquentes, pourvu que l'on sorte des archives de la Société. Les articles de la presse locale qui relatent les concours organisés par la Société tempèrent l'efficacité de ses mots d'ordre. Âl'évidence, les mutations esthétiques induites par le rapprochement de l'art et de l'industrie heurtent certains observateurs. L'équilibre entre dynamique nationale et identités régionales est difficile à trouver. Les divergences dans les appréciations esthétiques se doublent, s'agissant de la formation, de la nécessité d'adaptation aux besoins économiques locaux.

L'économie des honneurs vient couronner des formations techniques et des productions de qualité, en remaniant la tradition du chef-d'œuvre au temps du multiple industriel. Cette économie de la qualité repose toutefois sur un va-et-vient constant entre l'individuel et le collectif, entre une pédagogie par la production de l'objet de qualité et une pédagogie centrée sur l'exposition de cet objet produit.

\section{Le temps de l'exposition : une pédagogie de l'objet de qualité}

Les projets récompensés par la Société d'encouragement sont exposés successivement, de juillet à novembre chaque année, dans dix-neuf villes de province, à raison de huit jours par ville. Ce concours n'est donc pas seulement l'occasion d'un classement entre de jeunes artistes: il suscite des jugements esthétiques, notamment dans les procès-verbaux $\mathrm{du}$ jury ou les rapports annuels du secré- taire général. L'exposition itinérante des travaux donne de bons résultats à en croire ce dernier: «les presses locales s'y intéressent vivement, forçant l'attention des municipalités et d'un grand nombre de personnes jusque-là indifférentes à l'enseignement du dessin et à son développement: c'est ainsi que nous avons contribué à la récente ouverture d'un cours de composition décorative à 
l'école des beaux-arts de Montpellier ${ }^{23}$.» Que ces expositions soient plus ou moins bien accueillies, que des critiques parfois virulentes apparaissent, de nombreux exemples le montrent. Un article du Courrier $d u$ Nord, apparemment rédigé par un professeur d'art décoratif de Tourcoing, est tout à fait révélateur. L'intérêt de ces expositions locales y est reconnu pour les différentes catégories de visiteurs:

«Les élèves y trouvent une émulation précieuse; le public s'y renseigne sur les tendances, les progrès ou les faiblesses de notre enseignement des arts appliqués; des artisans, fabricants, ferronniers, bronziers, sculpteurs-ornemanistes, façonniers, $\mathrm{y}$ trouvent parfois des indications pour le renouvellement de leurs modèles, l'interprétation originale de la flore, l'adaptation de la matière à des besoins nouveaux.»

Pourtant, l'uniformisation de la composition et des procédés est vigoureusement dénoncée:

"Que l'on ne vienne pas prétendre que des jeunes gens de 18 à 25 ans qui pour la plupart n'ont pas voyagé, ont à Marseille, à Lyon ou à Lille, des facultés, des tendances, des moyens, et jusqu'à des procédés identiques sans que rien ne les nivelle! [...] L'empereur Guillaume est, paraît-il, très fier d'avoir inculqué l'automatisme à son peuple. Messieurs les inspecteurs généraux sont capables des mêmes fiertés. Nous pas ${ }^{24}$.»

Ces résistances illustrent aussi les diverses prescriptions et définitions de la qualité utilisées, y compris parmi les membres de la Société. Les débats esthé- tiques comme les polémiques habituelles sur la centralisation et la décentralisation renvoient à la difficulté d'établir des normes communes de qualité. Les concours antérieurs organisés par la Société sont invoqués pour critiquer cette tendance à l'uniformisation. L'intérêt des expositions n'est pas en cause, ni l'économie des honneurs dont elles procèdent, mais bien les prescriptions de qualité transmises dans le cours de la formation technique puis dans l'exposition des projets et objets primés qui font débat. C'est aussi renvoyer à une voie française de l'industrialisation, telle qu'elle a pu être décrite comme protectrice de la qualité et du «sur-mesure» distincts de la production de masse ${ }^{25}$. L'exposition relève de la promotion d'une nouvelle «culture du travail» par des associations privées œuvrant en bonne intelligence avec les représentants de la République naissante.

Les expositions internationales des arts décoratifs et industriels modernes relèvent d'autres logiques, attachées à la promotion du «goût français» et d'un savoir-faire dont la continuité passe par la formation technique. La naissance et l'organisation de ces expositions s'étendent sur près d'une vingtaine $\mathrm{d}^{\prime}$ années ${ }^{26}$. Après $1^{\prime}$ Union centrale des arts décoratifs, la Société d'encouragement prend part au mouvement, en 1912 et 1913. Associée à la commission chargée par le ministère du Commerce et de l'Industrie de définir la forme de l'exposition envisagée, elle voit aussi deux de ses membres, son secrétaire général Georges-Roger Sandoz et l'historien d'art Jean Guiffrey, dresser la généalogie des arts appliqués et industries d'art aux expositions, afin de justifier le projet de 
cette exposition internationale des arts décoratifs modernes. Les témoignages font généralement défaut sur la réception de telles expositions, dont la fréquence - si on réunit les expositions universelles, nationales, régionales, locales, plus ou moins thématiques - et la fréquentation sont grandes. Sans doute peut-on y trouver les indices d'une pédagogie de l'objet de qualité, dont l'exposition est censée fournir les caractères $d u$ bon goût voire de la mode. Cette pédagogie se lit dans les rapports et jusque dans les dispositions qu'on dirait aujourd'hui muséographiques.

Les effets de l'action de la Société d'encouragement paraissent contrastés. Les aides et l'émulation que ses membres suscitent permettent d'encourager individuellement et collectivement les industries d'art. Cependant, elle semble accompagner plutôt que véritablement peser sur les transformations de la production et de la formation. Ni la guerre, ni le recours au taylorisme ne bouleversent l'action de la Société, qui semble se cantonner de plus en plus au soutien des industries de luxe.

Entre le modèle du métier - souvent idéalement ramené à la corporation fantasmée voire décriée au mépris de sa diversité - et les modalités de codification de la qualité du travail par les grilles de classification Parodi après 1945, il a existé une large place pour d'autres dispositifs de reconnaissance de la qualité du travail $^{27}$. Dans ce procès de reconnaissance, reconsidérer la place du produit induit aussi une réflexion sur la nostalgie et l'industrialisation. Dans les concours organisés par la Société, le projet demandé relève du paradigme du chef-d'œuvre adapté au temps de l'industrie. Aussi la qualité intervient-elle à plusieurs titres. Elle peut caractériser soit le producteur, qu'il se présente comme ouvrier d'art, artisan ou artiste, soit sa qualification définie en acte par son travail, soit le produit «fini». Elle fonctionne dans chaque cas comme une variable pertinente de différenciation. L'économie des honneurs, par sa fonction de hiérarchisation parmi des égaux, s'intègre par conséquent à une économie de la qualité décisive dans la montée de l'enjeu politique de la formation technique.

\section{Notes}

1. Stéphane Lembré est maître de conférences en histoire contemporaine à l'ESPE Université Lille Nord de France, membre du Centre de recherche et d'études Histoire et Sociétés. Ses travaux portent sur l'histoire de l'éducation et l'histoire économique et sociale. Il a publié récemment L'école des producteurs. Aux origines de l'enseignement technique en France (1800-1940) (Rennes, Presses universitaires de Rennes, 2013). Contact: slembre@gmail.com.

2. Antoine Prost, «La démocratisation: histoire d'une notion ", Éducation, société et politiques: une histoire de l'enseignement en France de 1945 à nos jours, $2^{\mathrm{e}}$ éd. Paris, Seuil, 1997, p. 47-62. Sur l'origine des honneurs et leur devenir: Olivier Ihl, «Gouverner par les honneurs. Distinctions honorifiques et économie politique dans l'Europe du début du XIX ${ }^{\mathrm{e}}$ siècle", Genèses, n 55 , juin 2004, p. 4-26.

3. Olivier Ihl, Le mérite et la République. Essai sur la société des émules, Paris, Gallimard, 2007, p. 221.

4. Whitney Walton, France at the Crystal Palace: bourgeois taste and artisan manufacture in the Nineteenth Century, Berkeley, University of California press, 1992.

5. Alessandro Stanziani (dir.), La qualité des produits en France. XVIII $-\mathrm{XX}^{e}$ siècles, Paris, Belin, 2003.

6. Cette étude se fonde sur les archives de la Société d'encouragement à l'art et à l'industrie (SEAI), déposées aux Archives nationales du monde du travail (désormais ANMT) sous la cote 38 AS. L'histoire en est retracée par Stéphane Laurent, Les arts appliqués en France. Genèse d'un enseignement, Paris, CTHS, 1999, p. 357-368.

7. Serge Benoît, Gérard Emptoz et Denis Woronoff (dir.), Encourager l'innovation en France et en 
Europe: autour du bicentenaire de la Société d'encouragement pour l'industrie nationale, Paris, CTHS, 2006; Serge Benoît, "Associer le développement artistique et l'innovation et promouvoir les arts industriels: une orientation majeure de la Société d'encouragement pour l'industrie nationale au XIX ${ }^{e}$ siècle", dans Pierre Lamard et Nicolas Stoskopf (dir.), Art $\mathcal{E}$ industrie XVIII ${ }^{e}-\mathrm{XXI} I^{e}$ siècle, Paris, Picard, 2013, p. 39-50.

8. Anne-Laure Carré, Marie-Sophie Corcy, Christiane Demeulenaere-Douyère et Liliane Hilaire-Pérez (dir.), Les expositions universelles en France au XIX siècle. Techniques Publics Patrimoines, Paris, CNRS Éditions, Collection Alpha, 2012.

9. Gérard Bodé et Philippe Savoie (dir.), L'offre locale d'enseignement: les formations techniques et intermédiaires, $\mathrm{XIX}^{e}-\mathrm{XX} \mathrm{X}^{e}$ siècles, Histoire de l'éducation, n 66, 1995; Stéphane Lembré, L'école des producteurs. Aux origines de l'enseignement technique 18001940, Rennes, Presses universitaires de Rennes, 2013.

10. Thérèse Charmasson, Anne-Marie Lelorrain, Yannick Ripa, L'enseignement technique de la Révolution à nos jours, t. I: 1789-1926, Paris, INRP, Economica, 1987.

11. ANMT 38 AS 1: historique de la Société d'encouragement à l'art et à l'industrie.

12. Georg Maag, "Les machines ne sont rien sans l'art. De l'union des arts et de l'industrie du comte de Laborde, et les réactions de la presse", Romantisme, 1983, vol. 13, 41, p. 41-56.

13. Genèse évoquée dans $l^{\prime}$ «Historique de la Société d'encouragement à l'art et à l'industrie 1889-1929», extrait du Bulletin, $\mathrm{n}^{\circ} 16,4^{\mathrm{e}}$ trimestre 1929.

14. Commission d'enquête sur la situation des ouvriers et des industries d'art, instituée par décret en date du 24 décembre 1881, Paris, Imprimerie A. Quantin, 1884. Le discours d'ouverture d'A. Proust identifie bien les enjeux. Voir Vincent Dubois, «Le ministère des Arts (1881-1882) ou l'institutionnalisation manquée d'une politique artistique républicaine», Sociétés $\mathcal{E}$ Représentations, 2001 (1), $\mathrm{n}^{\circ} 11$, p. 229-261.

15. Marius Vachon, La crise industrielle et artistique en France et en Europe, Paris, Libraire illustrée, 1886, p. 60.

16. Marie-Claude Genet-Delacroix, Art et État sous la III République. Le système des beaux-arts 1870-1940, Paris, Publications de la Sorbonne, 1992, p. 7.

17. ANMT, 38 AS 2: procès-verbaux, volume 1 , Historique de la Société.

18. AD Nord, 79 J 7: séance de la chambre de commerce de Roubaix du 29 décembre 1893.

19. ANMT, 38 AS 43: rapport du secrétaire général à l'assemblée générale annuelle du 18 décembre 1896. Voir Jérémie Cerman, Le papier peint Art nouveau: création, production, diffusion, Paris, Mare \& Martin, 2012.

20. ANMT, 38 AS 21: rapport sur le $49^{\mathrm{e}}$ concours général annuel de composition décorative et industrielle, 1939.

21. Renaud d'Enfert, L'enseignement $d u$ dessin en France: figure humaine et dessin géométrique, 1750-1850, Paris, Belin, 2003; Agnès Lahalle, Les écoles de dessin au XVIII siècle: entre arts libéraux et arts mécaniques, Rennes, Presses universitaires de Rennes, 2006.

22. Manuel Charpy, Le théâtre des objets. Espaces privés, culture matérielle et identité bourgeoise. Paris, 1830-1914, thèse de doctorat, Université François-Rabelais de Tours, 2010.

23. ANMT, 38 AS 43: rapport du secrétaire général à l'assemblée générale annuelle du 18 décembre 1896.

24. Le Courrier du Nord, 19 novembre 1912.

25. Alain Cottereau, «Problèmes de conceptualisation comparative de l'industrialisation: l'exemple des ouvriers de la chaussure en France et en Grande-Bretagne», dans Susanna Magri et Christian Topalov (dir.), Villes ouvrières, 1900-1950, Paris, L'Harmattan, 1990, p. 41-80.

26. Georges-Roger Sandoz et Jean Guiffrey, Arts appliqués et industries d'art aux Expositions, Paris, Comité français des expositions à l'étranger et Société d'encouragement à l'art et à l'industrie, Paris, 1912. Voir Simon Dell, «The consumer and the making of the Exposition internationale des arts décoratifs et industriels modernes, 19071925 », Journal of Design History, vol. 12, n 4, 1999, p. 311-325.

27. Steven Kaplan et Philippe Minard (dir.), La France, malade du corporatisme? XVIII $-\mathrm{XX}^{e}$ siècles, Paris, Belin, 2004; Michel Lallement, «Qualités du travail et critique de la reconnaissance", dans Alain Caillé (dir.), La quête de reconnaissance. Nouveau phénomène social total, Paris, La Découverte, 2007, p. 75-76. Sur le métier et son imaginaire, Yves Lequin, «Le métier», dans Pierre Nora (dir.), Les lieux de mémoire. Les France: Traditions, Paris, Gallimard, 1992, p. 377-419. 